\title{
Atividade antimicrobiana in vitro de quinupristina/dalfopristina para cocos gram-positivos isolados de cinco centros brasileiros: resultado do estudo de vigilância L-SMART
}

\author{
Antimicrobial in vitro activity of quinupristin/dalfopristin against gram-positive cocci isolated from 5 Brazilian \\ centers: results from the local smart (L-SMART) surveillance study
}

Caio Mendes ${ }^{1,2}$

Sumiko I. Sinto ${ }^{1,2}$

André Hsiung ${ }^{1,2}$

Carmen Oplustil ${ }^{1,2}$

Lúcia Teixeira ${ }^{3}$

Adília Segura ${ }^{4}$

Dilair Souza ${ }^{5}$

Afonso Barth ${ }^{6}$

Antonio Carlos Nicodemo ${ }^{1}$

\begin{tabular}{|c|c|}
\hline unitermos & esumo \\
\hline $\begin{array}{l}\text { Programas de vigilância } \\
\text { de resistência } \\
\text { Resistência a antimicrobianos } \\
\text { Cocos gram-positivos }\end{array}$ & 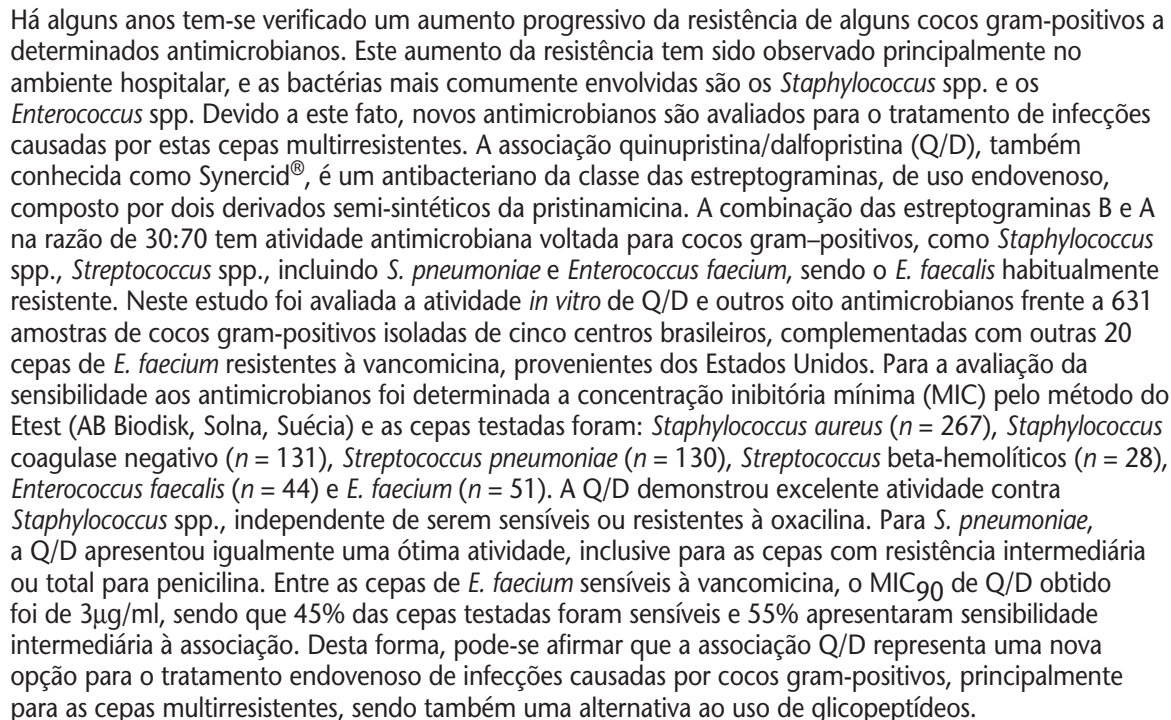 \\
\hline
\end{tabular}

abstract

A progressive increase of resistance among Gram-positive cocci (GPC) towards some antimicrobial agents has been observed for the past few years. This rise of resistance, most often seen in Staphylococcus spp., and Enterococcus spp., has mainly been noticed in hospital environments. Due to these recent patterns of resistance, newly developed antimicrobial agents need to be evaluated for the treatment of infections caused by these multi-resistant microorganisms. Quinupristin/dalfopristin (Q/D), also known as Synercid ${ }^{\circledR}$, is an antimicrobial agent of intravenous administration, composed of two semi-synthetic derivatives of pristinomycin belonging to the group of streptogramins. The combination of streptogramins $B$ and $A$ at 3:7 ratio has an antimicrobial activity against gram-positive cocci. This combination has potent activity against gram-positive cocci such as Staphylococcus spp., Streptococcus spp. including Streptococcus pneumoniae, and Enterococcus faecium. However, strains of $\mathrm{E}$. faecalis are usually resistant to this compound. The aim of this study was to evaluate the in vitro activity of $Q / D$ and other eight antibiotics against 631 strains of GPC isolated from five Brazilian centers. Additionally, 20 vancomycin-resistant strains of $\mathrm{E}$. faecium provided by a reference center from the United States were also included in this study. Minimal Inhibitory Concentrations (MICs) were determined by E-test methodology (AB Biodisk, Solna, Sweden), using standardized and controlled procedures.

The evaluated strains were as follows: Staphylococcus aureus (267), coagulase negative Staphylococcus

(131), Streptococcus pneumoniae (130), $\beta$-hemolytic Streptococcus (28), Enterococcus faecalis (44), and E. faecium (51). Quinuprintin/dalfopristin presented an excellent activity against Staphylococcus spp., regardless if these were susceptible or not to oxacillin. Against $\mathrm{S}$. pneumoniae, Q/D also presented excellent activitiy regardless of their susceptibility to penicillin. Among vancomycin susceptible E. faecium studied, the MIC 90 was $3 \mathrm{mg} / \mathrm{ml}$ where $45 \%$ of the strains were susceptible, and $55 \%$ revealed intermediate resistance to quinupristin/dalfopristin. Overall, Q/D showed good activity against Staphylococcus spp., Streptococcus spp. including S. pneumoniae, and Enterococcus faecium representing a new option for the treatment of infections caused by multi-resistant gram-positive cocci, as well as an alternative for the use of glycopeptides. key words

Surveillance resistance programs

Antimicrobial resistance

Gram-positive cocci
1. Laboratório de Investigação Médica - LIM 54 - HC/FMUSP. 2. Fleury - Centro de Medicina Diagnóstica.

3. Instituto de Microbiologia Universidade Federal do Rio de laneiro.

4. Laboratório Exame e Hospital de Base, Brasilia.

5. Hospital de Clínicas de Curitiba. 6. Hospital de Clínicas de Porto Alegre.

0 estudo LSMART foi possível devido ao suporte financeiro da Aventis Pharma (Brasil). 


\section{Introdução}

Os cocos gram-positivos são importantes agentes de infecção, e a resistência destes patógenos a antimicrobianos tem aumentado principalmente no ambiente hospitalar (2). Estes patógenos incluem Staphylococcus aureus, Staphylococcus coagulase-negativos (SCN), Enterococcus spp. e Streptococcus pneumoniae $(2,10)$.

Os Staphylococcus spp. oxacilina-resistentes geralmente também apresentam resistência a fluoroquinolonas, lincosamidas e macrolídeos, e a opção terapêutica atual são os glicopeptídeos. Porém há publicações em que se constata o isolamento de $S$. aureus oxacilina-resistente com sensibilidade reduzida a glicopeptídeos (GISA), tornando, desta forma, mais restritas as opções terapêuticas (5).

Entre os Enterococcus spp., há relatos de cepas resistentes à vancomicina em nosso meio $(3,11)$. Nos últimos anos, os enterococos têm emergido como importantes agentes de infecção hospitalar, tendo gerado sérios problemas devido ao desenvolvimento de resistência. Os E. faecium são geralmente menos suscetíveis aos antibióticos betalactâmicos e aminoglicosídeos, e as cepas de E. faecium resistentes a glicopeptídeos geralmente são resistentes a todos os outros antimicrobianos usualmente disponíveis (4).

Como conseqüência destas alterações no perfil de sensibilidade dos cocos gram-positivos, novos antimicrobianos estão sendo pesquisados e, neste estudo, avaliamos a atividade in vitro da $\mathrm{Q} / \mathrm{D}$ utilizando 651 cepas de cocos grampositivos como parte do programa de vigilância denominado Local Synercid ${ }^{\circledR}$ Microbiologic Assessment of Resistance Trends (L-SMART).

\section{Materiais e métodos}

No período de janeiro a outubro de 1999, cinco instituições de diferentes estados brasileiros (São Paulo, Rio de Janeiro, Rio Grande do Sul e Paraná), além do Distrito Federal, forneceram cepas de bactérias gram-positivas obtidas de casos clínicos para avaliação in vitro da atividade da Q/D e de outros oito antimicrobianos.

Coube a cada centro participante avaliar cerca de 150 cepas, sendo 30 de Staphylococcus aureus sensíveis à oxacilina, 20 de $S$. aureus resistentes à oxacilina, 15 de Staphylococcus coagulase-negativos oxacilina-sensíveis, 20 de Staphylococcus coagulase-negativos oxacilina-resistentes, dez de Enterococcus faecalis, 20 de Enterococcus faecium, 30 de Streptococcus pneumoniae e cinco cepas de Streptococcus beta-hemolíticos. Para complementar este estudo, um dos centros (LIM 54 - HC/FMUSP) testou adicionalmente 20 cepas de E. faecium resistentes à vancomicina, originárias dos Estados Unidos (Laboratório MRL).

Todas as cepas foram identificadas por espécie, de acordo com a metodologia preconizada pela Sociedade Americana de Microbiologia (7).

As cepas avaliadas eram provenientes de diversas amostras clínicas, como sangue, liquor, ossos e articulações, pele e estruturas da pele, líquido de cavidade abdominal, trato respiratório superior, trato respiratório inferior e outros.

Para a avaliação da resistência à $Q / D$ e a outros antimicrobianos, foi utilizado o método do Etest (AB Biodisk Solna, Suécia) para todas as cepas, com o inóculo ajustado na escala 0,5 de McFarland. Todas as placas foram incubadas a $35^{\circ} \mathrm{C}$ em estufa aeróbia, por 18 a 24 horas, exceto as placas de Streptococcus spp., que foram incubadas em estufa com $5 \%$ de $\mathrm{CO}_{2}$. Os resultados foram interpretados utilizando-se os critérios interpretativos do método da microdiluição sugeridos pelo National Committee for Clinical Laboratory Standards (9).

O controle de qualidade foi realizado com cepas de Staphylococcus aureus ATCC 29213, Enterococcus faecalis ATCC 29212 e Streptococcus pneumoniae ATCC 49619, toda vez que o teste da avaliação da resistência aos antimicrobianos era realizado.

Para Staphylococcus spp. as drogas avaliadas foram: quinupristina/dalfopristina, oxacilina, vancomicina, teicoplanina, ciprofloxacina, clindamicina, eritromicina, gentamicina, penicilina e sulfametoxazol/trimetoprim.

Para Enterococcus spp. as drogas avaliadas foram: quinupristina/dalfopristina, vancomicina, teicoplanina, ciprofloxacina, ampicilina, penicilina, gentamicina highlevel e estreptomicina high-level.

Para Streptococcus pneumoniae as drogas avaliadas foram: quinupristina/dalfopristina, vancomicina, clindamicina, eritromicina, sulfametoxazol/trimetoprima, cloranfenicol, penicilina e cefotaxima.

Para Streptococcus beta-hemolíticos as drogas avaliadas foram: quinupristina/dalfopristina, vancomicina, clindamicina, eritromicina, cloranfenicol, penicilina e cefotaxima.

\section{Resultados}

As 651 cepas avaliadas estavam assim distribuídas: 267 Staphylococcus aureus, 131 Staphylococcus coagulase-nega- 
tivos, 130 Streptococcus pneumoniae, 28 Streptococcus betahemolíticos, 44 Enterococcus faecalis, 31 Enterococcus faecium vancomicina-sensíveis e 20 E. faecium vancomicinaresistentes.

Entre os S. aureus e Staphylococcus coagulase-negativos, respectivamente $44,6 \%$ e $58 \%$ eram resistentes à oxacilina, e o $\mathrm{MIC}_{90}$ obtido nas cepas de $S$. aureus oxacilina-resistentes e oxacilina-sensíveis para Q/D foi de $0,5 \mu \mathrm{g} / \mathrm{ml}$. Para os Staphylococcus coagulase-negativos oxacilina-resistentes e oxacilina-sensíveis, o $\mathrm{MIC}_{90}$ obtido para Q/D foi de $0,75 \mu \mathrm{g} / \mathrm{ml}$ e $0,38 \mu \mathrm{g} / \mathrm{ml}$, respectivamente, com $100 \%$ de sensibilidade para ambas as espécies, conforme demonstrado nas Tabelas $\mathbf{1}$ e $\mathbf{2}$.

Do total das 130 cepas de S. pneumoniae, 95 (73\%) eram sensíveis à penicilina, 31 (24\%) apresentavam nível intermediário de resistência e quatro (3\%) apresentavam resistência total à penicilina. $\mathrm{O} \mathrm{MIC}_{90}$ obtido para $\mathrm{Q} / \mathrm{D}$ para as cepas de S. pneumoniae foi de $1 \mu \mathrm{g} / \mathrm{ml}$, mesmo para as cepas totalmente resistentes à penicilina, com $100 \%$ de sensibilidade. Para eritromicina e clindamicina, as cepas de S. pneumoniae, com MIC intermediário à penici- lina, apresentaram 19\% e $11 \%$ de resistência, respectivamente, conforme demonstrado na Tabela 3.

Entre as cepas de Streptococcus beta-hemolíticos, 11\% apresentaram resistência à eritromicina, e houve $100 \%$ de sensibilidade para $Q / D$, vancomicina, betalactâmicos, clindamicina e cloranfenicol, conforme Tabela 4.

Entre os E. faecalis, 100\% das cepas apresentaram sensibilidade aos glicopeptídeos e à ampicilina, sendo que para Q/D e penicilina as cepas apresentaram resistência de $80 \%$ e $11 \%$ respectivamente, conforme Tabela 5 .

Entre as cepas de E. faecium resistentes à vancomicina, obtivemos um $\mathrm{MIC}_{90}$ de $2 \mu \mathrm{g} / \mathrm{ml}$, com $70 \%$ de sensibilidade para Q/D e 30\% com resistência intermediária, sendo que $90 \%$ destas cepas foram resistentes a ampicilina e penicilina, conforme demonstrado na Tabela 6.

Entre as cepas de E. faecium sensíveis a vancomicina e teicoplanina, o $\mathrm{MIC}_{90}$ obtido para Q/D foi de $3 \mu \mathrm{g} / \mathrm{ml}$, com $45 \%$ de sensibilidade e $55 \%$ de resistência intermediária. A sensibilidade com relação à ampicilina e à penicilina foi de $74 \%$ e $65 \%$ respectivamente, como mostrado na Tabela 7.

Atividade in vitro de quinupristina/dalfopristina (Q/D) e outros antimicrobianos frente a

Tabela 1 Staphylococcus aureus oxacilina-resistentes e sensiveis isolados de cinco centros brasileiros

\begin{tabular}{llccccc}
\hline Microrganismo & Antimicrobiano & MIC $_{50}$ & MIC $_{90}$ & Range & $\%$ S & $\%$ R \\
S. aureus & Ciprofloxacina & $>32$ & $>32$ & $0,125->32$ & 5 & 93 \\
oxacilina-R & Clindamicina & $>256$ & $>256$ & $0,032->256$ & 4 & 96 \\
$n=119$ & Eritromicina & $>256$ & $>256$ & $0,19->256$ & 2 & 98 \\
& Gentamicina & $>256$ & $>256$ & $0,25->256$ & 6 & 94 \\
& Oxacilina & $>256$ & $>256$ & $4->256$ & 0 & 100 \\
& Penicilina & 48 & 128 & $3->256$ & 0 & 100 \\
& SXT & $>32$ & $>32$ & $0,064->32$ & 16 & 84 \\
& O/D & 0,38 & 0,5 & $0,094-1$ & 100 & 0 \\
S. aureus & Teicoplanina & 1,5 & 2 & $0,125-4$ & 100 & 0 \\
oxacilina-S & Ciprofloxacina & 0,19 & 0,38 & $0,064->32$ & 98 & 1 \\
$n=148$ & Clindamicina & 0,064 & 0,125 & $<0,016->256$ & 97 & 3 \\
& Eritromicina & 0,19 & 12 & $0,047->256$ & 78 & 14 \\
& Gentamicina (L) & 0,25 & 0,75 & $0,047->256$ & 95 & 5 \\
& Oxacilina & 0,25 & 0,5 & $0,032-2$ & 100 & 0 \\
& Penicilina & 1,5 & 6 & $<0,016->256$ & 7 & 93 \\
& SXT & 0,047 & 0,094 & $0,008->32$ & 98 & 2 \\
& O/D & 0,38 & 0,5 & $0,094-0,75$ & 100 & 0 \\
& Teicoplanina & 1 & 2 & $0,125-6$ & 100 & 0 \\
& Vancomicina & 1 & 1,5 & $0,25-3$ & 100 & 0 \\
\hline
\end{tabular}

SXT = sulfametoxazol/trimetoprim; $S=$ sensivel; $R=$ resistente. 
Atividade in vitro de quinupristina/dalfopristina (Q/D) e outros antimicrobianos frente a Staphylococcus coagulase-negativos (SCN) oxacilina-resistentes e sensiveis isolados de cinco Tabela 2 centros brasileiros

\begin{tabular}{llccccc}
\hline Microrganismo & Antimicrobiano & MIC $_{50}$ & MIC $_{90}$ & Range & $\%$ S & $\%$ R \\
SCN & Ciprofloxacina & 0,5 & $>32$ & $0,047->32$ & 58 & 37 \\
oxacilina-R & Clindamicina & 0,125 & $>256$ & $<0,016->256$ & 58 & 40 \\
& Eritromicina & 12 & $>256$ & $0,032->256$ & 39 & 59 \\
& Gentamicina & 16 & $>256$ & $0,032->256$ & 32 & 65 \\
& Oxacilina & 16 & $>256$ & $0,5->256$ & 0 & 100 \\
& Penicilina & 8 & $>256$ & $0,032->256$ & 4 & 96 \\
& SXT & 3 & $>32$ & $0,023->32$ & 44 & 56 \\
O/D & 0,25 & 0,75 & $0,064-1$ & 100 & 0 \\
SCN & Teicoplanina & 2 & 8 & $0,016-48$ & 96 & 4 \\
$n=55$ & Vancomicina & 1,5 & 2 & $0,5-3$ & 100 & 0 \\
& Ciprofloxacina & 0,094 & 0,19 & $0,032->32$ & 98 & 2 \\
& Clindamicina & 0,064 & 0,125 & $<0,016->256$ & 98 & 2 \\
& Eritromicina & 0,125 & 4 & $<0,016->256$ & 89 & 9 \\
& Gentamicina & 0,094 & 24 & $<0,064-64$ & 89 & 9 \\
& Oxacilina & 0,125 & 0,25 & $0,032-0,25$ & 100 & 0 \\
& Penicilina & 0,125 & 1 & $<0,016->256$ & 60 & 40 \\
& SXT & 0,094 & 32 & $0,008->32$ & 89 & 11 \\
& O/D & 0,25 & 0,38 & $0,094-0,5$ & 100 & 0 \\
& Teicoplanina & 0,75 & 4 & $0,032-4$ & 100 & 0
\end{tabular}

SXT = sulfametoxazol/trimetoprim; $S$ = sensível; $R$ = resistente.

Com relação aos aminoglicosídeos (high-level), 100\% das cepas de $E$. faecium e $E$. faecalis foram inibidas pela estreptomicina (independente das cepas serem sensíveis ou resistentes à vancomicina), e entre as cepas de $E$. faecalis, $82 \%$ foram inibidas pela gentamicina high-level, conforme Tabela 5. Para as amostras de E. faecium vancomicinaresistentes e sensíveis, $65 \%$ e $80 \%$ das cepas foram inibidas pela gentamicina high-level, como demonstrado nas Tabelas 6 e 7.

\section{Conclusão e discussão}

Diferentemente de outros inibidores da síntese de proteínas, como os macrolídeos e lincosaminas, que são inativados na presença dos genes erm, a associação Q/D pode determinar um efeito sinérgico e eficaz mesmo contra aqueles cocos gram-positivos que apresentam resistência cruzada aos antimicrobianos do grupo MLS: macrolídeos, lincosaminas e estreptogramina B (8).

Neste estudo, encontramos $45 \%$ de sensibilidade a $\mathrm{Q} / \mathrm{D}$ entre as cepas de $E$. faecium sensíveis à vancomicina e 70\% de sensibilidade frente a cepas de E. faecium resistentes à vancomicina, não havendo resistência total em nenhuma das amostras testadas.

O percentual de sensibilidade obtido entre as cepas sensíveis à vancomicina difere dos obtidos no grupo de estudo GSMART para a América Latina, onde foram relatados $80 \%$ de sensibilidade a Q/D para as cepas de $E$. faecium sensíveis à vancomicina e apenas $12 \%$ de sensibilidade $\mathrm{a} \mathrm{Q} / \mathrm{D}$ para as cepas resistentes à vancomicina (11).

Do total das cepas de E. faecium sensíveis à vancomicina $(n=31), 55 \%$ apresentaram resistência intermediária a $\mathrm{Q} / \mathrm{D}$, com $\mathrm{MIC}_{50}$ e $\mathrm{MIC}_{90}$ de $1,5 \mu \mathrm{g} / \mathrm{ml}$ e $3 \mu \mathrm{g} / \mathrm{ml}$, respectivamente, sendo que 13 destas 31 cepas foram isoladas na cidade do Rio de Janeiro. A maioria das cepas que demonstraram resistência intermediária apresentou MICs no valor de $1,5 \mu \mathrm{g} / \mathrm{ml}$ e $2 \mu \mathrm{g} / \mathrm{ml}$.

É importante salientar que, neste estudo, nenhuma cepa se mostrou resistente a Q/D, e outro estudo, o Sentry, também detectou na América Latina, no Canadá e nos Estados Unidos, taxas de sensibilidade à Q/D de 66,7\%, $89,4 \%$ e $92,3 \%$ respectivamente (10). 


\section{Atividade in vitro de quinupristina/dalfopristina (Q/D) e outros antimicrobianos frente a}

Tabela 3 Streptococcus pneumoniae isolados de cinco centros brasileiros

\begin{tabular}{|c|c|c|c|c|c|c|}
\hline Microrganismo & Antimicrobiano & $\mathrm{MIC}_{50}$ & $\mathrm{MIC}_{90}$ & Range & $\% S$ & $\% \mathbf{R}$ \\
\hline \multirow{8}{*}{$\begin{array}{l}\text { S. pneumoniae } \\
\text { penicilina-S } \\
n=95\end{array}$} & Cefotaxima & 0,023 & 0,125 & $0,016-0,32$ & 100 & 0 \\
\hline & Clindamicina & 0,064 & 0,125 & $0,016->256$ & 97 & 2 \\
\hline & Cloranfenicol & 1,5 & 2 & $0,125-6$ & 100 & 0 \\
\hline & Eritromicina & 0,047 & 0,094 & $0,016->256$ & 98 & 2 \\
\hline & Penicilina & 0,016 & 0,032 & $0,006-0,064$ & 100 & 0 \\
\hline & SXT & 0,75 & 16 & $0,016->32$ & 49 & 27 \\
\hline & Q/D & 0,5 & 1 & $0,125-1$ & 100 & 0 \\
\hline & Vancomicina & 0,38 & 0,75 & $0,032-1$ & 100 & 0 \\
\hline \multirow{8}{*}{$\begin{array}{l}\text { S. pneumoniae } \\
\text { penicilina-I } \\
n=31\end{array}$} & Cefotaxima & 0,25 & 1 & $0,064-1,5$ & 78 & 0 \\
\hline & Clindamicina & 0,094 & 0,25 & $0,023->256$ & 89 & 11 \\
\hline & Cloranfenicol & 1,5 & 2 & $0,19-8$ & 97 & 3 \\
\hline & Eritromicina & 0,064 & 3 & $0,023->256$ & 81 & 19 \\
\hline & Penicilina & 0,25 & 1 & $0,064-1,5$ & 0 & 0 \\
\hline & SXT & 4 & $>32$ & $0,25->32$ & 11 & 68 \\
\hline & Q/D & 0,5 & 1 & $0,19-1$ & 100 & 0 \\
\hline & Vancomicina & 0,38 & 0,5 & $0,125-1$ & 100 & 0 \\
\hline \multirow{7}{*}{$\begin{array}{l}\text { S. pneumoniae } \\
\text { penicilina-R } \\
n=4\end{array}$} & Cefotaxima & 1 & 1,5 & $0,75-1,5$ & 0 & 25 \\
\hline & Clindamicina & $>256$ & $>256$ & $0,032->256$ & 25 & 75 \\
\hline & Cloranfenicol & 2 & 8 & $0,5-8$ & 75 & 25 \\
\hline & Eritromicina & 4 & $>256$ & $1,5->256$ & 25 & 75 \\
\hline & Penicilina & 1,5 & 2 & $1,5-2$ & 0 & 100 \\
\hline & SXT & 24 & 32 & $12-64$ & 0 & 100 \\
\hline & Q/D & 0,25 & 1 & $0,125-1$ & 100 & 0 \\
\hline Vancomicina & 0,38 & 0,38 & $0,125-0,5$ & 100 & 0 & \\
\hline
\end{tabular}

SXT = sulfametoxazol/trimetoprim; $S$ = sensível; $R$ = resistente; I = intermediário.

Atividade in vitro de quinupristina/dalfopristina (Q/D) e outros antimicrobianos frente a Tabela 4 Streptococcus beta-hemolíticos isolados de cinco centros brasileiros

$\begin{array}{llccccc}\text { Microrganismo } & \text { Antimicrobiano } & \text { MIC }_{50} & \text { MIC }_{90} & \text { Range } & \% \mathrm{~S} & \% \mathrm{R} \\ \text { Streptococcus } & \text { Cefotaxima } & 0,023 & 0,047 & 0,006-0,125 & 100 & 0 \\ \text { beta-hemolíticos } & \text { Clindamicina } & 0,064 & 0,125 & 0,023-0,19 & 100 & 0 \\ n=28 & \text { Cloranfenicol } & 2 & 3 & 0,5-4 & 100 & 0 \\ & \text { Eritromicina } & 0,064 & 4 & 0,032-8 & 89 & 11 \\ & \text { Penicilina } & <0,016 & 0,023 & <0,016-0,032 & 100 & 0 \\ & \text { Q/D } & 0,25 & 0,5 & 0,094-0,75 & 100 & 0 \\ & \text { Vancomicina } & 0,25 & 0,75 & 0,19-1 & 100 & 0\end{array}$

$\mathrm{S}$ = sensível; $\mathrm{R}$ = resistente. 


\section{Tabela 5 Enterococcus faecalis isolados de cinco centros brasileiros}

\begin{tabular}{llccccc}
\hline Microrganismo & Antimicrobiano & MIC $_{50}$ & MIC $_{90}$ & Range & $\% \mathrm{~S}$ & $\% \mathrm{R}$ \\
E. faecalis & Ampicilina & 0,75 & 3 & $0,023-6$ & 100 & 0 \\
$n=44$ & Ciprofloxacina & 0,75 & $>32$ & $0,25->32$ & 73 & 23 \\
& Estreptomicina (HL) & 128 & $>256$ & $1,5->256$ & 100 & 0 \\
& Gentamicina (HL) & 12 & $>1.024$ & $<0,064->1.024$ & 82 & 18 \\
& Penicilina & 1,5 & 16 & $0,032-24$ & 89 & 11 \\
& Q/D & 12 & $>32$ & $0,5->32$ & 5 & 80 \\
& Teicoplanina & 0,25 & 0,5 & $0,047-1,5$ & 100 & 0 \\
& Vancomicina & 1,5 & 2 & $0,38-3$ & 100 & 0 \\
\hline
\end{tabular}

$\mathrm{S}=$ sensível; $\mathrm{R}=$ resistente; $\mathrm{HL}=$ high-level.

Atividade in vitro de quinupristina/dalfopristina (Q/D) e outros antimicrobianos frente a Tabela 6 Enterococcus faecium não-pertencentes a isolados brasileiros

$\begin{array}{llccccc}\text { Microrganismo } & \text { Antimicrobiano } & \text { MIC }_{50} & \text { MIC }_{90} & \text { Range } & \% \text { S } & \% \text { R } \\ \text { E. faecium } & \text { Ampicilina } & 32 & 256 & 1->256 & 10 & 90 \\ \text { vancomicina-R } & \text { Ciprofloxacina } & 1,5 & 3 & 0,5->32 & 45 & 10 \\ n=20 & \text { Estreptomicina (HL) } & >256 & >256 & 24->256 & 100 & 0 \\ & \text { Gentamicina (HL) } & 128 & 1.024 & 2->1.024 & 65 & 35 \\ & \text { Penicilina } & >256 & >256 & 2-256 & 10 & 90 \\ & \text { Q/D } & 0,75 & 2 & 0,5-2 & 70 & 0 \\ & \text { Teicoplanina } & 1,5 & 32 & 0,25-64 & 60 & 15 \\ & \text { Vancomicina } & >256 & >256 & 32->256 & 0 & 100\end{array}$

$\mathrm{S}=$ sensível; $\mathrm{R}=$ resistente; $\mathrm{HL}=$ high-level.

\section{Atividade in vitro de quinupristina/dalfopristina (Q/D) e outros antimicrobianos frente a}

Tabela 7

\section{Enterococcus faecium isolados de cinco centros brasileiros}

\begin{tabular}{llccccc}
\hline Microrganismo & Antimicrobiano & MIC $_{50}$ & MIC $_{90}$ & Range & $\% \mathrm{~S}$ & $\% \mathbb{R}$ \\
E. faecium & Ampicilina & 2 & 128 & $0,064->256$ & 74 & 26 \\
vancomicina-S & Ciprofloxacina & 1 & 3 & $0,25-4$ & 55 & 10 \\
$n=31$ & Estreptomicina (HL) & 128 & $>256$ & $0,38->256$ & 100 & 0 \\
& Gentamicina (HL) & 8 & $>1.024$ & $0,125->1.024$ & 80 & 20 \\
& Penicilina & 4 & $>256$ & $0,094->256$ & 65 & 34 \\
& Q/D & 1,5 & 3 & $0,23-3$ & 45 & 0 \\
& Teicoplanina & 0,38 & 0,75 & $0,047-1,5$ & 100 & 0 \\
& Vancomicina & 0,5 & 1,5 & $0,25-2$ & 100 & 0 \\
\hline
\end{tabular}


As 20 cepas de $E$. faecium vancomicina-resistentes aqui analisadas mostraram $70 \%$ de sensibilidade à $Q / D$, sendo que as restantes mostraram resistência intermediária, e, neste grupo de amostras com resistência intermediária, a maioria apresentou MICs de 1,5 $\mathrm{g} / \mathrm{ml}$ (cepas com resistência intermediária encontram-se na faixa $>1 \mu \mathrm{g} / \mathrm{ml} \mathrm{a}<4 \mu \mathrm{g} / \mathrm{ml}$ ).

Os resultados aqui obtidos são relevantes, pois também outros relatos têm mostrado bons resultados clínicos e microbiológicos em pacientes com infecções por $E$. faecium vancomicina-resistentes, quando tratados com $Q / D(6)$.

Entre as cepas de $E$. faecalis ( $n=44)$, verificaram-se $100 \%$ de sensibilidade à ampicilina, e $89 \%$ de sensibilidade à penicilina. Entre os E. faecium sensíveis à vancomicina, $74 \%$ apresentaram sensibilidade à ampicilina, e $26 \%$ mostraram-se resistentes, havendo um aumento no percentual de resistência (10\%) em relação aos encontrados pelo estudo GSMART (11).

A Q/D apresentou excelente atividade in vitro para cepas de S. aureus e Staphylococcus coagulase-negativos, mes- mo entre as cepas oxacilina-resistentes, com 100\% de sensibilidade. A Q/D apresentou também excelente atividade in vitro para as cepas de S. pneumoniae ( $100 \%$ de sensibilidade), inclusive para as cepas resistentes à penicilina.

Para as cepas de Streptococcus beta-hemolíticos $(n=28)$, a $Q / D$ apresentou igualmente uma ótima atividade in vitro, com $\mathrm{MIC}_{50}$ e $\mathrm{MIC}_{90}$ de $0,25 \mu \mathrm{g} / \mathrm{ml} \mathrm{e}$ $0,5 \mu \mathrm{g} / \mathrm{ml}$, respectivamente.

Com base neste estudo e em outros estudos in vitro (1, $10,11)$, pode-se concluir que a associação Q/D representa uma nova opção para o tratamento endovenoso de infecções causadas por cocos gram-positivos multirresistentes, sendo também uma opção ao uso de glicopeptídeos.

\section{Agradecimentos}

Agradecemos a valiosa contribuição de todos os centros participantes e a Thais Cocarelli pela ajuda na análise estatística dos resultados.

\section{Referências}

I. Chang, S.C. et al. In vitro activity of quinupristin/dalfopristin against clinical isolates of common gram-positive bacteria in Taiwan. Diag. Microbiol. Infect. Dis., 33: 299-303, 1999.

2. Cormican, M.G. \& Jones, R.N. Emerging resistance to antimicrobial agents in gram-positive bacteria. Drugs, 5I (suppl. I): 6- I2, 1996.

3. Dalla Costa, L.M. et al. Vancomycin-resistant Enterococcus faecium: first case in Brazil. Bras. J. Infect. Dis. (BJID), 2(3): 160-3, 1998.

4. Eliopoulos, G.M. et al. Characterization of vancomycin-resistant Enterococcus faecium isolates from the United States and their susceptibility in vitro to dalfopristin-quinupristin. Antimicrob. Agents Chemother., 42: I088-92, 1998.

5. Hiramatsu, K. et al. Methicillin-resistant Staphylococcus aureus clinical strains with reduced vancomycin susceptibility. J.Antimicrob. Chemother., 40: 135-6, 1997.

6. Moellering, R.C. et al. The efficacy and safety of quinupristin/ dalfopristin for treatment of infections caused by vancomycin-resistant Enterococcus faecium. Antimicrob. Agents Chemother., 44:251-61, 1999.

7. Murray, P.R. et al. Manual of Clinical Microbiology. 6.ed. Washington, DC:American Society for Microbiology, 1995.

8. Nadler, H. et al. Quinupristin/dalfopristin: a novel selectivespectrum antibiotic for the treatment of multi-resistant and other gram-positive pathogens. Clin. Microbiol. Newsletter, 2I (13): I03-12, 1999.
9. National Committee for Clinical Laboratory Standards (NCCLS) Performance standards for antimicrobial susceptibility testing: nineth informational supplement, MI0-S9. Wayne, PA, 1999.

10. Pfaller, M. et al. Survey of blood stream infections attributable to Gram-positive cocci: antimicrobial susceptibility of isolates collected in 1997 in the United States, Canada, and Latin America from the Sentry Antimicrobial Surveillance Program. Diag. Microbiol. Infect. Dis., 33: 283 97, 1999.

I I. Sader, H.S. et al. Antimicrobial susceptibility of quinupristin/ dalfopristin tested against Gram-positive cocci from Latin America: results from the Global SMART (GSMART) surveillance study. Bras.J. Infect. Dis. (B/ID), 5( I):21-31,200 I.
Endereço para correspondência

Caio Mendes

Seção de Microbiologia Fleury - Centro de Medicina Diagnóstica Av. General Waldomiro de Lima 508 CEP 04344-070 - São Paulo-SP e-mail: caio.mendes@fleury.com.br 